\title{
Should We Be CHARMed?
}

\author{
Colombel JF, Sandborn WJ, Rutgeerts $P$, et al. Adalimumab for maintenance of clinical response and \\ remission in patients with Crohn's disease: the CHARM Trial. Gastroenterology. 2007;132:52-65.
}

A dalimumab, a subcutaneously administered human antiTNF- $\alpha$ monoclonal antibody, has recently been approved by the FDA for the treatment of Crohn's disease (CD). The CHARM Trial was a multicenter clinical trial of the efficacy and safety of adalimumab for the maintenance of disease remission in moderate to severe $\mathrm{CD}$.

Colombel et al reported the results of this randomized, double-blind, placebo-controlled study conducted at 92 sites in the US, Canada, Europe, Australia, and South Africa. Enrolled patients had moderate to severely active CD (Crohn's disease activity index [CDAI] 220-450) and included patients on stable doses of and azathioprine (AZA), 6-mercaptopurine (6-MP), methotrexate (MTX), 5-aminosalicylic acid (5-ASA), sulfasalazine, prednisone, or budesonide. Patients who had taken infliximab in the past were included if: 1) their last dose was more than 12 weeks prior and 2) they had an initial good response to infliximab. Notable exclusion criteria included patients with an ostomy or a history of recent or extensive small bowel resection.

All enrolled patients received adalimumab $80 \mathrm{mg}$ subcutaneously at week 0 followed by adalimumab $40 \mathrm{mg}$ subcutaneously at week 2 . At week 4 , patients were assessed for response (decrease $\mathrm{CDAI} \geq 70$ from baseline) to adalimumab induction. Subjects were then randomized to placebo, adalimumab $40 \mathrm{mg}$ every other week (qow), or adalimumab $40 \mathrm{mg}$ weekly (qw) with treatment lasting through week 56. The co-primary outcomes were the percent of week 4-randomized responders who were in clinical remission (defined as CDAI $<150$ ) at 1) week 26 and 2) week 56. Several secondary outcomes were explored and these included corticosteroidfree remission rates and fistula closure rates.

Of 854 patients enrolled, 76 withdrew prior to randomization, 499 (58\%) responded to induction and were randomized with 170 assigned to placebo, 172 assigned to adalimumab qow, and 157 assigned to adalimumab qw. Of the randomized responders, $12 \%, 36 \%$, and $41 \%$ of patients assigned to placebo, qow adalimumab, and qw adalimumab, respectively, were in clinical remission at week 56 . The differences between each adalimumab arm and the placebo

Received for publication April 25, 2007; accepted April 30, 2007. Copyright (C) 2007 Crohn's \& Colitis Foundation of America, Inc. DOI 10.1002/ibd.20191

Published online 13 June 2007 in Wiley InterScience (www.interscience. wiley.com). arm were statistically significant, but the differences between the adalimumab schedules were not significant.

The secondary endpoint of corticosteroid-free remission was reported for randomized responders initially on steroids. At week 56, 6\%, 29\%, and $23 \%$ of subjects taking placebo, qow adalimumab, and weekly adalimumab, respectively, had achieved steroid-free remission. Adalimumab was also reported to be superior to placebo for fistula closure. Of the subjects with fistulae, $30 \%$ of the pooled patients taking adalimumab compared with $13 \%$ taking placebo demonstrated fistula closure at week 26 with continued response at week 56.

The most commonly reported adverse event was CD exacerbation. This occurred at a significantly higher rate in the placebo arm (32.2\%) than among adalimumab-treated patients (19.6\% for every other week and $18.7 \%$ for every week). Injection site reactions, urinary tract infection (UTI), fatigue and headache occurred more commonly in 1 or both of the adalimumab groups as compared with placebo. Serious infectious adverse events were noted to be uncommon and not significantly different between treatment and placebo groups $(2.7 \%$ and $3.4 \%$, respectively). However, 2 cases of tuberculosis were identified, both in patients treated with adalimumab with negative purified protein derivatives (PPDs) and chest x-rays at study entry.

\section{COMMENT}

The CHARM trial was a randomized, double-blind, placebo-controlled study with concealed allocation in which an intention-to-treat analysis was conducted, and all enrolled patients were accounted for (those lost to follow-up, those who withdrew from the study, those who switched to openlabel therapy, and those who did not have CDAI assessments at weeks 26 and 56 were all considered failures to maintain remission).

One omission in the presentation of the results is that the reader is left to assume that randomization to the 3 arms truly did produce unbiased groups. The authors report the pooled baseline characteristics of the patients who were randomized, but do not present the baseline characteristics of the subjects in each of the 3 treatment arms. While bias is relatively unlikely to occur in the randomization of 499 subjects to 3 arms, it is possible that unanticipated baseline differences (i.e., in prior anti-TNF- $\alpha$ use) could have an impact on the results. This is highlighted by the primary 
efficacy analysis, which is adjusted for different rates of previous anti-TNF- $\alpha$ therapy in different study arms, although the reader is not informed of what those rates were.

The primary aim of this trial was to determine the maintenance rate of remission in patients treated with adalimumab who had a good initial response to induction therapy and received continued maintenance dosing. The pooled week 56 remission rate among initial responders treated with adalimumab was $38.6 \%$, compared with $11.7 \%$ for placebo. This difference yields a pooled number needed to treat (NNT) of 4 initial responder patients who would need to be maintained with adalimumab to achieve 1 extra week 56 remission compared to placebo (exact NNT 3.71, 95\% confidence interval [CI] 2.93-5.06).

The patient population studied in this trial had a motley treatment history. While nearly $50 \%$ had previously used a TNF- $\alpha$ antagonist, only $47 \%$ were on any immunosuppressive agent (including AZA, 6-MP, and MTX), 39\% were on 5 -ASAs, and $44 \%$ were on corticosteroids. This shows that real-world practice is far from standardized in either a step-up or a top-down algorithm, and it suggests that adalimumab may be efficacious for a wide range of patients.

One might expect that subjects with delayed infliximab failure might be unresponsive to adalimumab, but both TNFantagonist-naïve and TNF-antagonist-experienced patients on adalimumab had significantly greater maintenance of remission versus placebo. The TNF-antagonist-naïve patients did have higher rates of remission versus those with delayed infliximab failure (45\% versus $32 \%$ in the pooled adalimumab-treated patients), and this difference was statistically significant (benefit ratio 1.39, 95\% CI 1.05-1.84, $P=0.02$ ). Clinicians will have success with adalimumab in patients who have had delayed infliximab failure, but they should expect to have somewhat greater success rates in infliximab-naïve patients.

How does adalimumab maintenance compare to other immune modulators used for Crohn's disease? It is impossible to make definitive comparisons between therapies evaluated in different studies (due to differences in study patient characteristics, study design, and the timing and definition of the endpoints), but these results are not very different from those reported for infliximab in ACCENT I, which had a week 30 maintenance of remission NNT of $5 .{ }^{1}$ Similarly, the NNT for MTX from Feagan et al.'s study of maintenance of remission $^{2}$ was 4 (exact NNT 3.83, 95\% CI 2.09-22.81), and the Cochrane Review of thiopurine maintenance for $\mathrm{CD}$ calculates a NNT of $7 .{ }^{3}$ These are rather similar results for 4 immune system-modifying agents in CD. Each of these medications has its own risks, including serious infection and cancer, and these have to be carefully weighed by physicians and patients. These medications also differ substantially in cost, with 56-week medication costs from Drugstore.com ${ }^{4}$ of
US\$260 for methotrexate, US\$557 for azathioprine, US\$19,107 for adalimumab, and US\$16,438 for infliximab before infusion costs. Physicians and patients have to judge the balance between risks, benefits, and costs when choosing between these 4 medications. The large cost differential and similar maintenance efficacy suggest that we should consider MTX and AZA before using biologics.

The real-world effectiveness of adalimumab for maintenance is hard to estimate from a controlled clinical trial, but patients and clinicians want a reasonable estimate of the likelihood of success before they start a therapy, to help them weigh the benefits against the risks and costs. For every 100 subjects started on adalimumab in this study (pooling the results from both adalimumab schedules), 9 dropped out before 4 weeks, and 33 had a poor response at 4 weeks. Of the remaining 58 patients with a good week 4 response who continued in the maintenance phase, only 22 were in remission at week 56, compared to 7 with placebo. This is clearly superior to placebo, but the overall rate of remission at 1 year is relatively low. This illustrates how far we still have to go in the treatment of CD. Given the cost of adalimumab, methods to prospectively identify the 22 patients out of 100 most likely to achieve sustained remission (perhaps via immunogenetic profiling) would be very helpful for future clinical decision-making. Adalimumab appears to be a reasonably effective, reasonably well-tolerated, and quite expensive treatment option for CD. Adalimumab is an important addition to our limited armamentarium against $\mathrm{CD}$, and gives us a good option when infliximab fails. Having infliximab as our only biologic option in $\mathrm{CD}$ is reminiscent of the words of Nigel Tufnel (referring to guitar amplifiers that only go up to 10 in This Is Spinal Tap): "Where can you go from there? Nowhere." Adalimumab now gives us another anti-TNF- $\alpha$ option.

Dahlia Awais, MD

Peter D.R. Higgins, MD, PhD, MSc

Division of Gastroenterology

University of Michigan

Ann Arbor, Michigan

\section{REFERENCES}

1. Hanauer SB, Feagan BG, Lichtenstein GR, et al. Maintenance infliximab for Crohn's disease: the ACCENT I randomised trial. Lancet. 2002;359: 1541-1549.

2. Feagan BG, Fedorak RN, Irvine EJ, et al. A comparison of methotrexate with placebo for the maintenance of remission in Crohn's disease. North American Crohn's Study Group Investigators. N Engl J Med. 2000;342: $1627-1632$.

3. Timmer A, McDonald JW, Macdonald JK. Azathioprine and 6-mercaptopurine for maintenance of remission in ulcerative colitis. Cochrane Database Syst Rev 2007:CD000478.

4. www.drugstore.com. Prescription Price Checker. Accessed April 24, 2007. 